\title{
SPECTRAL AND STATISTICAL ANALYSIS OF FLOW-INDUCED VIBRATIONS
}

\author{
JIANGNAN LU ${ }^{1} \&$ J. M. NIEDZWECKI ${ }^{2}$ \\ ${ }^{1}$ Ocean Engineering Department, Texas A\&M University, College Station. \\ ${ }^{2}$ Department of Civil and Environmental Engineering and of the Ocean Engineering Department, \\ Texas A\&M University, College Station.
}

\begin{abstract}
A general approach that utilizes both spectral and extremal statistical methods are utilized to investigate the time series of flow-induced response behavior of a flexible horizontal cylinder subject to both random waves and constant current conditions. The cylinder model was $29 \mathrm{~m}$ long and had a slenderness ratio of approximately 760 . The random waves were generated using a JONSWAP wave amplitude spectrum. In addition, for some tests, the cylinder was towed at two different speeds to simulate the combined loading of random waves and constant current conditions. The data were initially analyzed using standard spectral analyses to interpret the cylinder's flow-induced response behavior and relate the findings to traditional deterministic parameters. Further analyses were performed using a generalized extreme value (GEV) distribution procedure that involved dividing the time series into blocks and fitting the block maxima of the extreme values in the measured response time series data. The Anderson-Darling (AD) test criterion and quantile plots were then used to assess whether the GEV distribution provides a satisfactory fit to the data capturing the statistical characteristics in the flexible cylinder's flow-induced response behavior, which was stochastic in nature. For the data set analyzed, the extremal GEV methodology presented was observed to provide excellent results for the random wave cases and moderately good-to-good results for the combined random wave and constant current cases.

Keywords: combined loading, currents, flexible horizontal cylinder, flow-induced response, GEV extremal statistics, random waves, spectral analysis.
\end{abstract}

\section{INTRODUCTION}

Long flexible cylindrical structures such as marine risers and horizontal pipelines are widely used in the offshore industry. Their flow-induced vibrations when exposed to combined random wave and current loadings are very complicated and have been the focus of numerous experimental investigations reported in the open literature. Vandiver [1] investigated the vortex-induced vibration of long flexible cylinders from numerous field experiments that he conducted and, in the process, identified important dimensionless parameters associated with predicting the observed response behavior. These parameters included the shear fraction $\Delta V / V_{\max }$, the number of potentially excited modes $N_{s}$, the mass ratio $m / \rho_{f} D^{2}$, and the mass-damping parameter $S_{G}=r \omega /\left(\rho_{f} V^{2}\right)$. The expression of the mass-damping parameter $S_{G}$ was first formulated by Griffin et al. [2] to predict the maximum response amplitude of flexible structures and cables in water. However, Sarpkaya [3, 4] reported that this parameter $S_{G}$ has many shortcomings, while Zdravkovich [5] suggested that $S_{G}$ was only valid for cylinders with a very high value of mass ratio. To address the limitations of this traditional mass-damping parameter, Vandiver [6] introduced a revised damping parameter $c^{*}=2 c \omega / \rho U^{2}$ and it was found valid at all frequencies where synchronization occurs between the wake and cylinder motion, disregarding the value of the mass ratio.

A more recently studied parameter that is closely related to flexible cylinder's VIV response amplitude is the Reynolds number Re. Klamo et al. [7] were the first to report that the peak 
value of VIV response amplitude will increase as $R e$ becomes large, while Swithenbank et al. [8] later provided the data sets of the dimensionless VIV amplitude $A$ / $D$ vs. $R e$ based on both laboratory and field test programs. Sometimes later, Resvanis et al. [9] investigated the trends between $R e$ and flexible marine riser's VIV response amplitude based on the experimental data obtained by Klamo [7], Govardhan and Williamson [10], and Shell's model basin test program [11]. They observed that for $R e$ between $5 \times 10^{3}$ and $2.2 \times 10^{5}$, the riser exhibited a trend to experience larger amplitudes in both in-line and cross-flow directions, but there also existed significant scatter between the predicted trends and the measured data.

A very important characteristic of flexible cylinder's VIV response behavior is its stochastic nature, but few relevant research studies could be found in the literature while most of the current popular VIV prediction programs in the industry were deterministic as reported by Resvanis and Vandiver in 2017 [12]. By introducing a short-duration moving window and calculating the mean and standard deviation of RMS response amplitude $A$ / $D$ within each window, Resvanis and Vandiver [12] were able to illustrate the statistical characteristics of the non-stationary VIV data obtained from Shell [11]. They concluded from their observations that future research effort is required to quantify the variability in flexible cylinder's VIV response behavior.

The purpose of this research study is to investigate a general approach to capture the statistical characteristics of the extreme values in the flexible horizontal cylinder's flow-induced response amplitudes in-line with the flow direction. The experimental data were obtained in an industrial scale model basin test program [13]. In this study, a horizontal cylinder was pretensioned on both sides and tested under a combination of random waves and steady current speeds achieved by towing the model at a constant speed in the wave basin. The measured cylinder's mid-span displacement was selected for the analysis in each of the test run and was first examined using spectral analysis to characterize the response behavior and relate it to traditional deterministic parameters. Then, a more general approach based on an extremal statistics methodology was utilized to investigate the statistical characteristics of the measured data. As part of this approach, the performance of the statistical models for each data set was assessed by goodness-of-fit test criterion that is widely used in statistical analyses.

\section{EXPERIMENTAL SETUP}

An industrial scale model basin test program was performed at Offshore Technology Research Center (OTRC) located at Texas A\&M University to investigate the flow-induced vibrations of a slender horizontal cylinder subjected to a combination of random waves and current loadings [13]. A schematic of the test set up is illustrated in Fig. 1. The model scale was determined to be 1:16 in accordance with the Froude scaling law. The length of the test cylinder was approximately $29 \mathrm{~m}$ and the outside and inside diameter were $38 \mathrm{~mm}$ and $22 \mathrm{~mm}$, respectively. Thus, the slenderness ratio $L / D$ was estimated to be 760 . The test cylinder provided by Fiberspar was constructed using a hollow plastic core wrapped with a composite material. The unit weight of the cylinder was $10.18 \mathrm{~N} / \mathrm{m}$, and the corresponding mass ratio was 0.91 . The cylinder was submerged at a water depth of $0.61 \mathrm{~m}$ and a pretension of 2277.5 $\mathrm{N}$ was applied to both ends of the model through the two vertical I-beams, which were rigidly fixed on the OTRC bridge. Since the young's modulus of the composite cylinder was unknown, Chitwood [13] was only able to provide an estimated range of the pre-tensioned cylinder's natural frequencies of vibration at different modes when subject to a uniform current or regular waves based on various assumptions. During the test, six biaxial accelerometers were placed inside the cylinder, and they were kept dry by sealing the ends of the cylinder and 


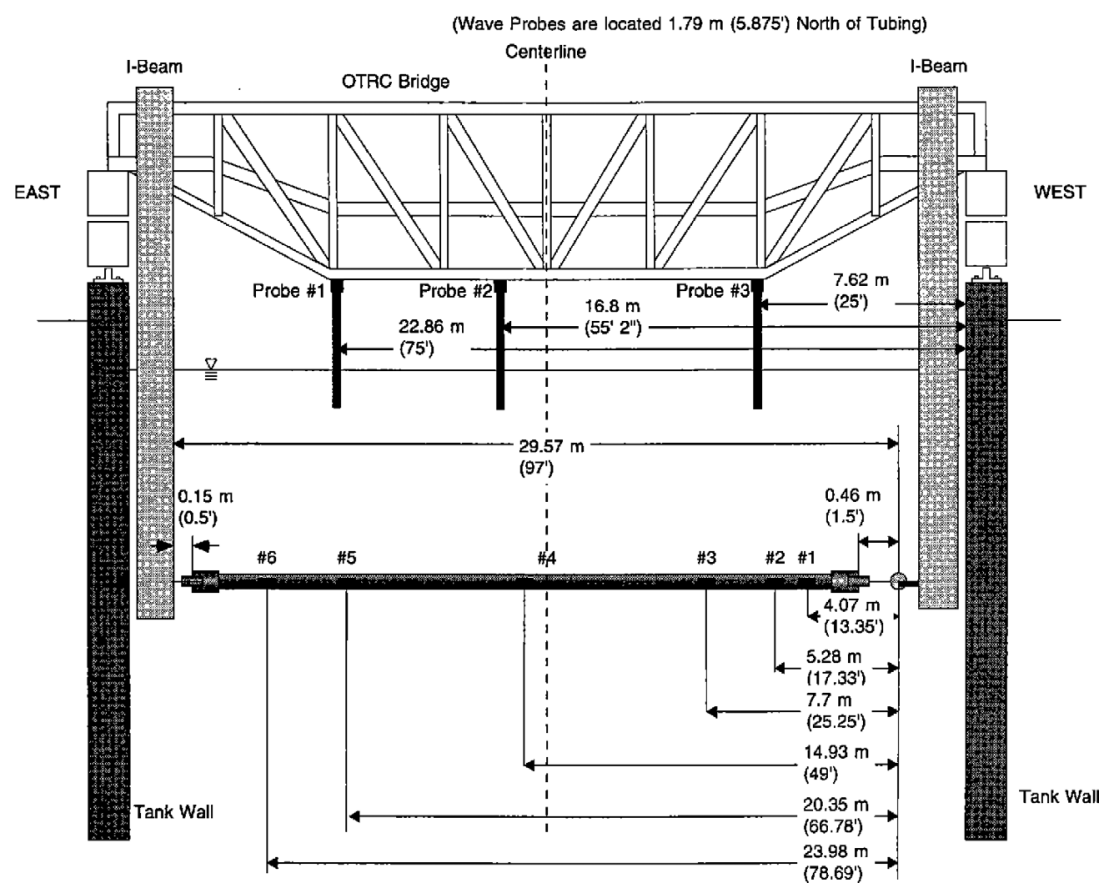

Figure 1: Schematic of the horizontal cylinder in the model basin (Chitwood [13]).

maintaining a slight pressure inside the cylinder model. Three wave probes were fixed on the bridge to measure the water surface elevation at $7.62 \mathrm{~m}$ west of the centerline, $1.58 \mathrm{~m}$ east of the centerline, and $7.62 \mathrm{~m}$ east of the centerline, respectively. The cylinder's mid-span displacement was measured at a time interval $\Delta t$ of $0.025 \mathrm{~s}$ using a string-potentiometer that was mounted on the bridge.

In this study, the model random sea conditions were generated using a JONSWAP wave amplitude spectrum with a significant wave height of $H_{S}=0.43 \mathrm{~m}$ and a peak period of $T_{p}=3 \mathrm{~s}$. For each unidirectional random wave test, the instrumented test cylinder was tested for $300 \mathrm{~s}$ and for the multi-directional random waves that had a 20-degree spreading angle each test lasted for $600 \mathrm{~s}$. As part of the test program, the cylinder model was towed at two different speeds during the random wave excitation. Due to the length of the model basin, the duration of each test was reduced. For the test where the towing speed was $0.244 \mathrm{~m} / \mathrm{s}$, the test lasted for only $60 \mathrm{~s}$, and when the towing speed was increased to $0.366 \mathrm{~m} / \mathrm{s}$, the duration was further reduced to $40 \mathrm{~s}$.

\section{METHODOLOGY}

\subsection{Spectral analysis}

Spectral analysis is a widely used technique to study the measured time series $X(t)$ in the frequency domain. The power spectral density function, $S(f)$, which is the Fourier transform of the autocorrelation function of $X(t)$, presents the energy distributions of the time series $X(t)$ at each frequency. The expression of the $i^{t h}$ spectral moment was given by Nigam [14] as follows: 


$$
\lambda_{i}=\int_{0}^{\infty} f^{i} S(f) d f
$$

where $f$ is the frequency in $\mathrm{Hz}$ and the average frequency $\bar{f}$ could be obtained by the expression

$$
\bar{f}=\lambda_{1} / \lambda_{0}
$$

To describe dispersion of $S(f)$ with respect to its central frequency, Nigam [14] defined a dimensionless spectral parameter in the following:

$$
q=\sqrt{1-\frac{\lambda_{1}^{2}}{\lambda_{0} \lambda_{2}}}
$$

In addition, the bandwidth of the process $X(t)$ could be shown by a bandwidth parameter $\varepsilon$ introduced by Cartwright and Longuet-Higgins [15] as

$$
\varepsilon=\sqrt{1-\frac{\lambda_{2}^{2}}{\lambda_{0} \lambda_{4}}}
$$

The above spectral parameters can be useful for one to understand the characteristics of the process $X(t)$ measured in the time domain. However, when the process $X(t)$ is highly stochastic, the values of these spectral parameters might vary significantly even under the same test conditions. In this case, it might no longer be feasible to characterize the process by a set of deterministic parameters and one might need to seek alternatives to capture the global response behavior of $X(t)$.

\subsection{Extremal statistics methods and goodness-of-fit assessment techniques}

The extremal statistics methods combined with the goodness-of-fit assessment techniques could provide a basis to capture the statistical characteristics of the measured process $X(t)$. This section presents a brief introduction of the theoretical background of the generalized extreme value (GEV) distribution and the Anderson-Darling goodness-of-fit test criterion based on the research work by Coles [16] and D'Agostino and Stephens [17].

\section{Generalized extreme value (GEV) distribution}

For a sequence of independent random variables $X_{1}, X_{2}, \ldots X_{n}$, that were measured consecutively at a fixed time interval of $\Delta t$, the maximum value of this process over the duration of $n \Delta t$ is

$$
M_{n}=\max \left\{X_{1}, X_{2}, \ldots, X_{n}\right\}
$$

where the probability that $M_{n}$ is less than or equal to $z$ could be expressed as

$$
P\left(M_{n} \leq z\right)=F(z)^{n}
$$

where $F$ is the distribution function shared by the sequence $X_{1}, X_{2}, \ldots, X_{n}$. Since the function $F$ is unknown, Coles [16] shifted his attention to look for approximated expressions of $F^{n}$ based only on the extreme values in the measured data. However, in his investigations, he observed that for any $z<z_{+}$, where $z_{+}$is the smallest value of $z$ such that $F(z)=1, F^{n}$ would 
decrease to zero at large values of $n$ and the distribution of $M_{n}$ would degenerate to a point mass on $z_{+}$. In Coles' formulations, this difficulty was solved with a linear renormalization on the expression of $M_{n}$ as

$$
M_{n}^{*}=\frac{M_{n}-b_{n}}{a_{n}}
$$

where $a_{n}$ and $b_{n}$ are a sequence of constants. Based on the extremal types theorem [16], one could obtain the following expression given $a_{n}>0$ and $b_{n}>0$

$$
P\left(\frac{M_{n}-b_{n}}{a_{n}} \leq z\right) \rightarrow G, \text { as } n \rightarrow \infty
$$

where $G$ is a non-degenerated distribution function that could be categorized into one of the three familiar extreme value distributions Gumbel, Fréchet, or Weibull, disregard the formulation of the distribution function $F$. Since each of the above three distributions has different forms of tail behavior, Coles [16] indicated that it is more appropriate to combine them as the following generalized extreme value (GEV) distribution

$$
G(z)=\exp \left\{-\left[1+\xi\left(\frac{z-\mu}{\sigma}\right)\right]^{-\frac{1}{\xi}}\right\}
$$

where $\xi$ is the shape parameter, $\mu$ is the location parameter, and $\sigma$ is the scale parameter. The distribution function $G(z)$ is defined on $\{z: 1+\xi(z-\mu) / \sigma>0)\}$ for $-\infty<\mu<+\infty, \sigma>0$, and $-\infty<\xi<\infty$. When the value of $\xi$ becomes zero, the function $G(z)$ simply reduces to the Gumbel distribution. This combined expression of the extreme value distributions makes it convenient for one to determine the most appropriate types of tail behavior by changing the value of $\xi$ based directly on the observed data [16]. In practice, given a series of independent observed values $\left\{X_{1}, X_{2}, \ldots,\right\}$ of a random process $X(t)$ that are measured at a time interval of $\Delta t$, one could divide it into $m$ blocks of equal length $n$ ( $n$ is large) to obtain a sequence of block maxima $\left\{M_{n, 1}, M_{n, 2}, \ldots, M_{n, m}\right\}$, where each element $M_{n, i}$ is the largest observed value within each block that has a length of $n \Delta t$. Then, the GEV distribution could be fitted to the sequence $\left\{M_{n, 1}, M_{n, 2}, \ldots, M_{n, m}\right\}$, and the fitted function could provide a basis to investigate the statistical characteristics of the extreme values in the measured time series $\left\{X_{1}, X_{2}, \ldots,\right\}$. What makes the GEV distribution more attractive is that even when the time series $\left\{X_{1}\right.$, $\left.X_{2}, \ldots,\right\}$ shows a temporal dependence and the extreme values occur in clusters, Coles [16] indicated that it is still valid to approximate the constructed blockmaxima $\left\{M_{n, 1}, M_{n, 2}, \ldots, M_{n, m}\right\}$ as independent random variables that could be properly fitted by the GEV distribution, as long as the long-range dependence at the extreme levels is weak. With this approximation, the GEV distribution could be utilized in a wide range of applications.

\section{Anderson-Darling (AD) test criterion}

Suppose $F_{0}$ is the proposed distribution function for a series of independent and identically distributed random variables $\left\{Y_{1}, Y_{2}, \ldots, Y_{n}\right\}$ that has a common continuous distribution function $F_{n}(y)$, Cramér and von Mises [18] formulated a criterion to assess the goodness of fit of $F_{0}$ compared to $F_{n}(y)$ as follows: 
Table 1: General guidelines for goodness-of-fit assessment based on the p-value.

\begin{tabular}{llllll}
\hline $\mathrm{p}$-value & $\mathrm{p}<0.01$ & $0.01<\mathrm{p}<0.05$ & $0.05<\mathrm{p}<0.15$ & $0.15<\mathrm{p}<0.25$ & $\mathrm{p}>0.25$ \\
\hline $\begin{array}{l}\text { Goodness } \\
\text { of fit }\end{array}$ & $\begin{array}{l}\text { Unaccept- } \\
\text { able }\end{array}$ & Poor & $\begin{array}{l}\text { Moderately } \\
\text { good }\end{array}$ & Good & Excellent \\
\hline
\end{tabular}

$$
Q_{n}=n \int_{-\infty}^{\infty}\left[F_{n}(y)-F_{0}(y)\right]^{2} \mathrm{w}(y) d F_{0}(y)
$$

where in the original Cramér-von Mises criterion the weighting function $w(y)$ is defined as unity. However, D'Agostino and Stephens [17] observed that this criterion could not satisfactorily detect the difference between $F_{0}$ and $F_{n}(y)$ in the tails of the distribution, which was usually the focus of extreme value analysis. To correct this weakness, Anderson and Darling [19] proposed a revised weighting function:

$$
\mathrm{w}(y)=\left[F_{0}(y)\left(1-F_{0}(y)\right)\right]^{-1}
$$

Introducing the revised weighting function to the original Cramér-von Mises criterion, the expression of the Anderson-Darling (AD) test criterion is obtained as follows:

$$
A_{n}^{2}=n \int_{-\infty}^{\infty} \frac{\left[F_{n}(y)-F_{0}(y)\right]^{2}}{\left[F_{0}(y)\left(1-F_{0}(y)\right)\right]} d F_{0}(y)
$$

The above revised test criterion is more sensitive to detect the discrepancies between $F_{0}$ and $F_{n}(y)$ in the tails of the distribution. In applications the quality of fit of the proposed model could be examined by the p-value of the AD test based on the generally accepted guidelines as shown in Table 1 .

\section{ANALYSIS OF THE MODEL BASIN DATA}

For the analyses that follow, the target variable was selected as the measured mid-span displacement $X(t)$. The power spectral densities were calculated based on Welch's method [20], and these results are used to initially interpret the cylinder's response behavior. Next, the GEV statistical methodology is used to investigate the characterization of the cylinder's response behavior observed in the experimental data.

\subsection{Spectral analysis of the cylinder's mid-span displacement}

Initially, the measured time series of the cylinder's mid-span displacement $X(t)$ and their power spectral density (PSD) calculated by Welch's method were shown in Figs 2-9. Then, both the statistical moments and the spectral moments of each test condition were calculated and presented in Table 2. In Table 2, the values of $\mu^{2}+\sigma^{2}$ were found to have a good agreement with the 0th spectral moment $\lambda_{0}$, which confirmed that the calculated PSD was correct.

When the cylinder was only subjected to unidirectional random waves, one observes that the time series plotted in Fig. 2 is very similar to a stationary random process. This observation is confirmed by examining the value of the skewness $\alpha_{3}$ and the kurtosis $\alpha_{4}$ as presented in Table 2, which only showed a small deviation from the Gaussian process. When the 


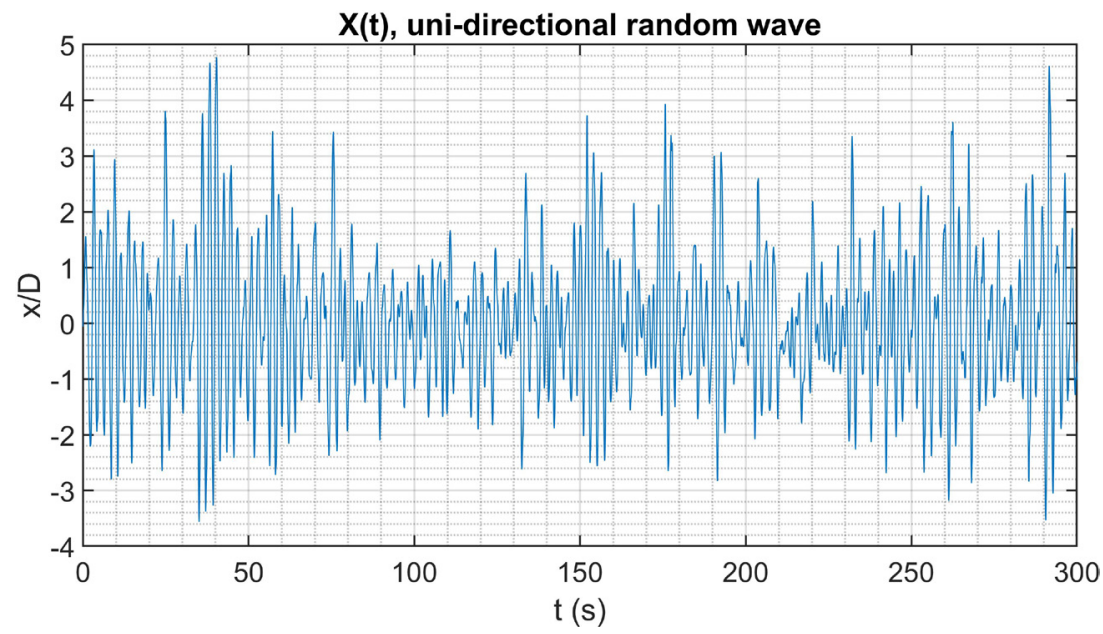

Figure 2: Time series of $X(t)$ under unidirectional random waves.

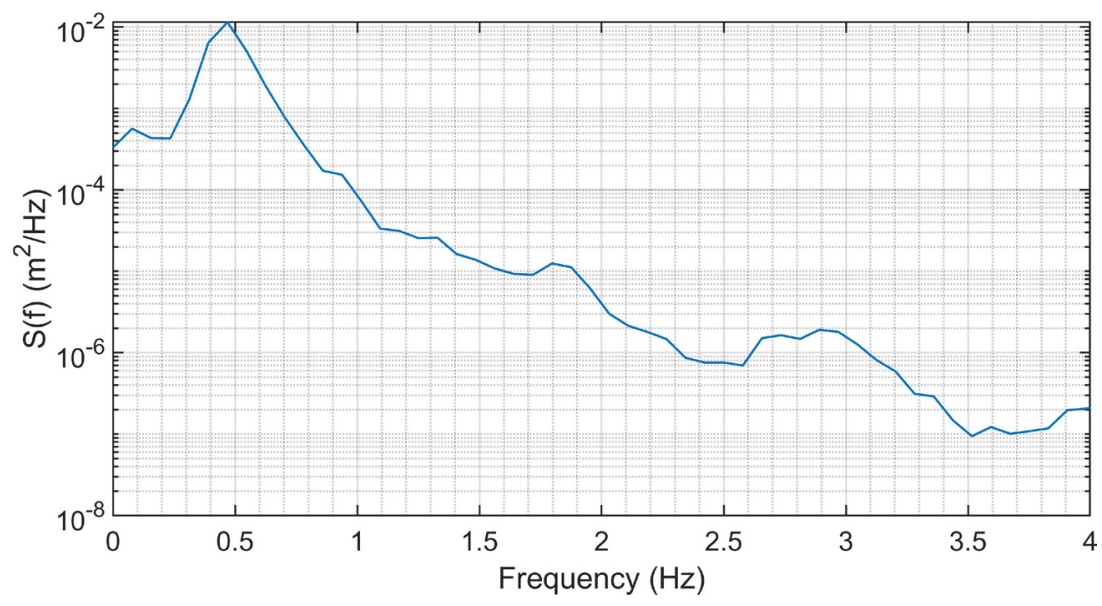

Figure 3: Power spectral density of $X(t)$ under unidirectional random waves.

random waves were generated in multi-directions with a 20-degree spreading angle, it is observed in Fig. 4 that the measured value of $X(t)$ exhibited significantly smaller amplitude while the statistical moments shown in Table 2 indicate that the process $X(t)$ is almost Gaussian.

For the random wave cases when a steady current of either $0.244 \mathrm{~m} / \mathrm{s}$ or $0.366 \mathrm{~m} / \mathrm{s}$ were introduced, a mean deflection was observed in the measured time series of $X(t)$ as presented in Figs 6 and 8. In Table 2, one observes that although the mean deflections of the four tests are relatively close, the higher-order statistical moments of each test show significant variations, especially when the current speed was increased to $0.366 \mathrm{~m} / \mathrm{s}$. These discrepancies might be attributed to the short duration of the test time and the complexity of the interactive response behavior of the cylinder subject to random waves combined with a steady current. In addition, the calculated mean frequency $\bar{f}$ was found to decrease from around $0.5 \mathrm{~Hz}$ to 


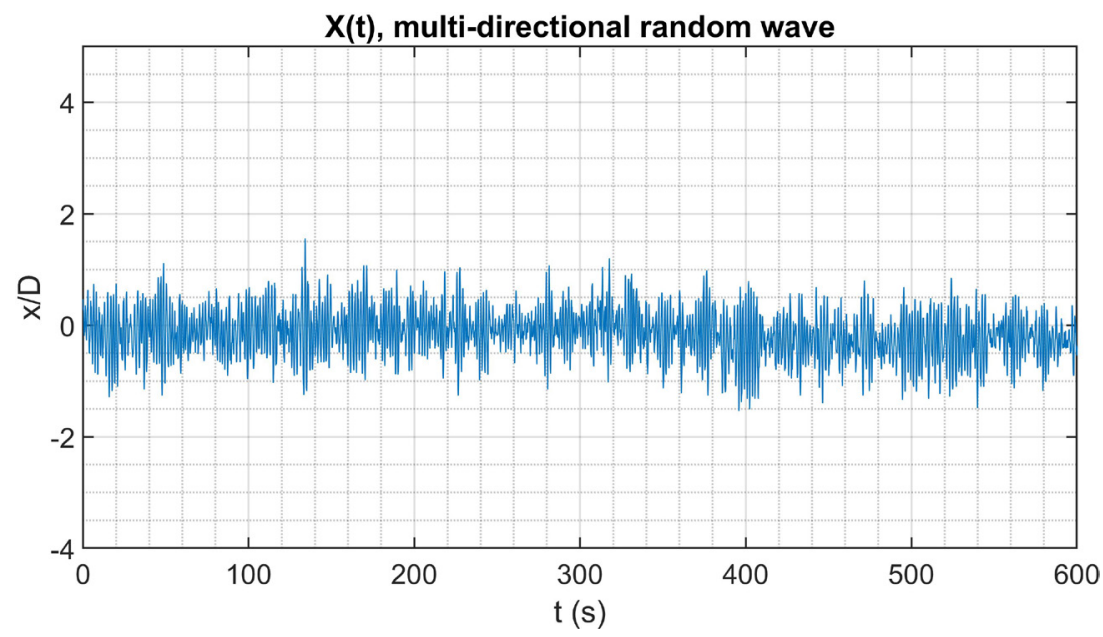

Figure 4: Time series of $X(t)$ under multi-directional random waves.

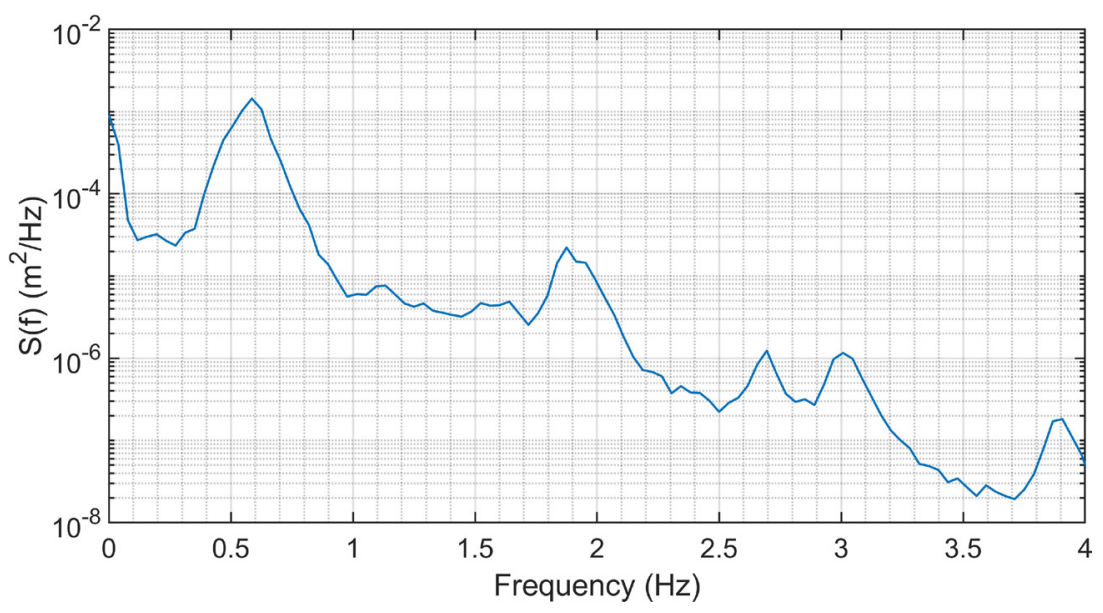

Figure 5: Power spectral density of $X(t)$ under multi-directional random waves.

below $0.1 \mathrm{~Hz}$ after the superposition of the current, while the expected VIV frequencies were not observed in the PSD plot as shown in Figs 7 and 9. This observation further illustrates the complexity of the cylinder's response behavior in these flow conditions.

Considering the significant variations in the calculated values of the traditional deterministic parameters and the complexity of the problem, one might conclude that it is extremely difficult to utilize a set of deterministic parameters to characterize the cylinder's flowinduced response behavior, which is stochastic in nature as addressed by Resvanis and Vandiver [12]. Thus, it becomes reasonable for one to acknowledge the stochastic nature of the cylinder's flow-induced motions and look for a general approach to investigate the statistical characteristics in the observed response behavior. In the following, an statistical approach based on generalized extreme value (GEV) distribution is utilized to analyze the cylinder's mid-span displacement $X(t)$ of all the test scenarios listed in Table 2. 

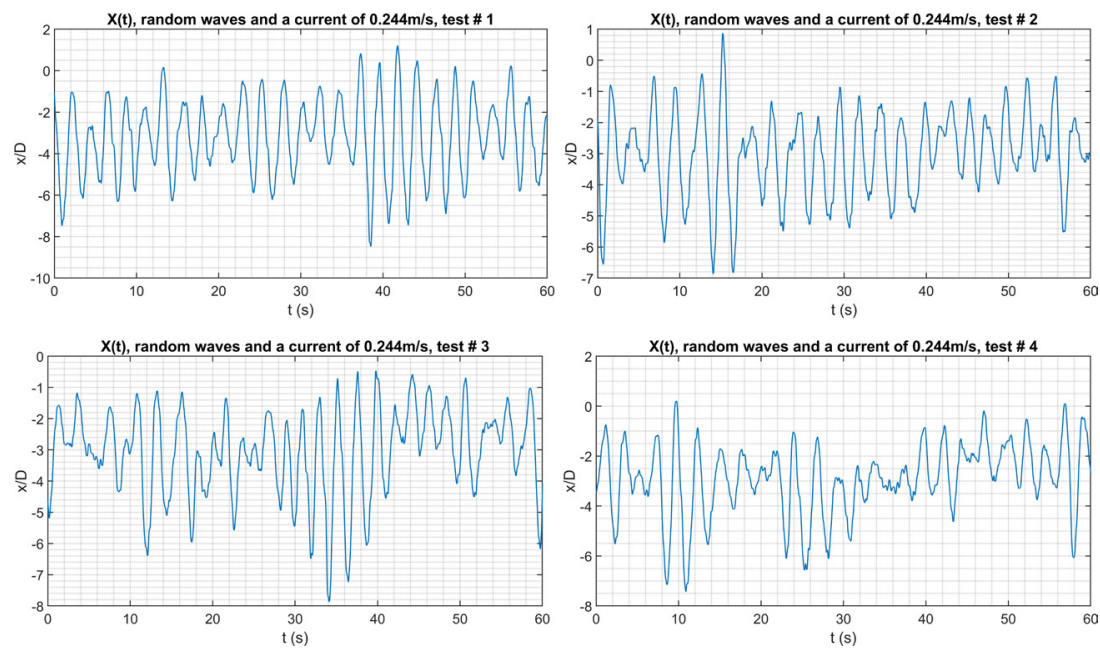

Figure 6: Time series of $X(t)$ under random waves and a current of $0.244 \mathrm{~m} / \mathrm{s}$.
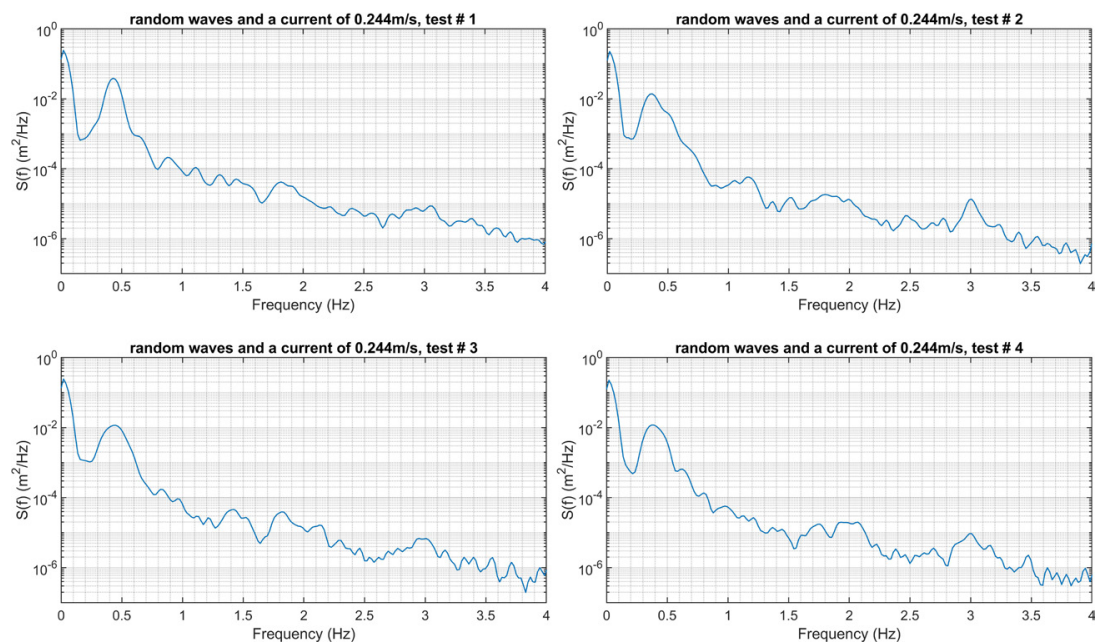

Figure 7: Power spectral density of $X(t)$ under random waves and a current of $0.244 \mathrm{~m} / \mathrm{s}$.

4.2 Statistical analysis of the cylinder's mid-span displacement

The statistical computing software package $\mathrm{R}$ [21] was used in this analysis. To analyze the data using the GEV distribution, one needs to first construct the sequence of block maxima $\left\{Z_{n, i}\right\}$ from the measured mid-span displacement $X(t)$. To select a proper value of the block size $n$, the time series plot under random waves in Figs 2 and 4 was zoomed in for the first 50 $\mathrm{s}$ and presented in Fig. 10. In Fig. 10, one observes that each oscillation cycle lasts for approximately $2 \mathrm{~s}$, which could also be found in Figs 6 and 8 when a steady current was superposed to the random waves. To make sure that the generated block maxima $\left\{Z_{n, i}\right\}$ could satisfactorily capture the extreme values of $X(t)$, the block size $n$ is determined to be 80 so each block 

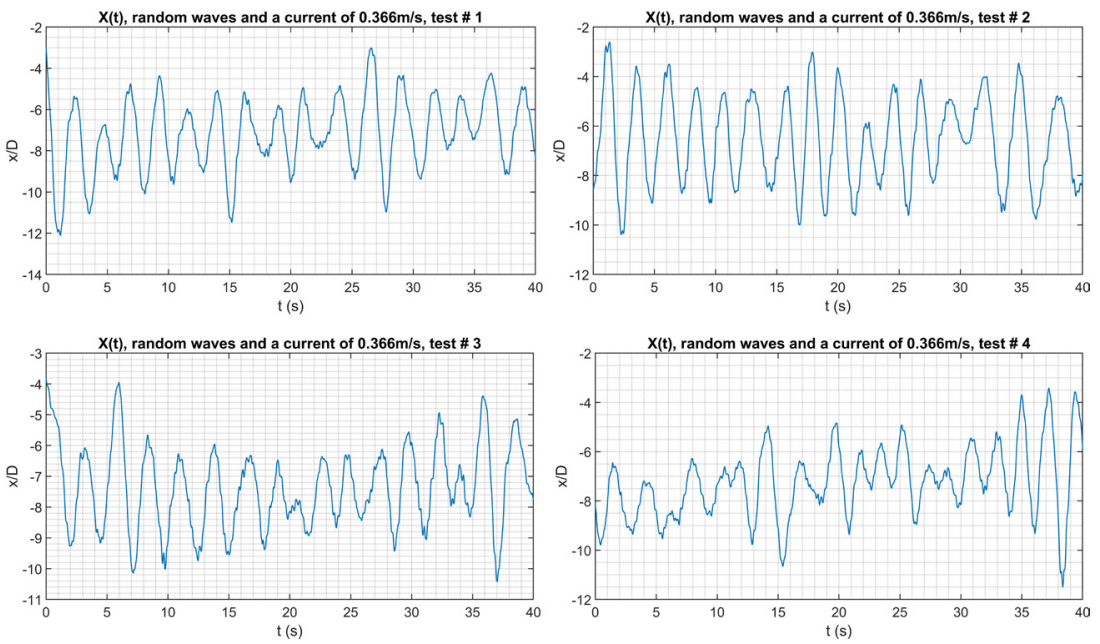

Figure 8: Time series of $X(t)$ under random waves and a current of $0.366 \mathrm{~m} / \mathrm{s}$.
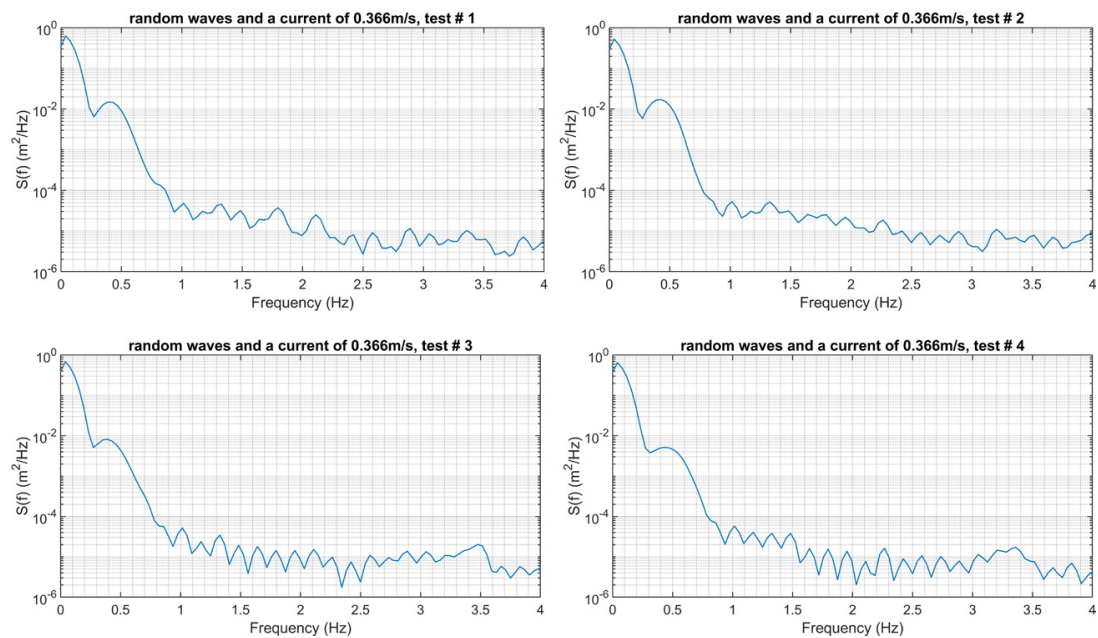

Figure 9: Power spectral density of $X(t)$ under random waves and a current of $0.366 \mathrm{~m} / \mathrm{s}$.

has a length of $n \Delta t=2 \mathrm{~s}$. Thus, each element $Z_{n, i}$ of block maxima would represent the extreme values observed in a complete oscillation cycle.

In Table 2, one observes that the mean value of $X(t)$ under random waves was almost 0 . Thus, the block maxima $Z_{n, i}$ was chosen as the observed maximum amplitude of each oscillation cycle for the first two cases. Later, when the cylinder was simultaneously subjected to random waves and a steady current, the mean value of $X(t)$ became negative due to the current. Thus, the block maxima $Z_{n, i}$ in these two cases was determined to be the maximum displacement in-line with the current direction recorded in a duration of 2 s. However, one also observes in Figs 6 and 8 that the duration of each test was relatively short and at most 20 complete oscillation cycles could be identified in each plot. Given such a short time series, one might not be able to obtain enough data points for extremal statistical analysis. To solve 
Table 2: Statistical and spectral moments of mid-span displacement $X(t)$.

\begin{tabular}{|c|c|c|c|c|c|c|c|c|c|}
\hline \multirow{2}{*}{$\begin{array}{l}\text { Param- } \\
\text { eters }\end{array}$} & $\mu$ & $\sigma$ & $a_{3}$ & $\alpha_{4}$ & $\mu^{2}+\sigma^{2}$ & $\lambda_{0}$ & $\bar{f}$ & $q$ & $\varepsilon$ \\
\hline & $\mathrm{m}$ & $\mathrm{m}$ & - & - & $\mathrm{m}^{2}$ & $\mathrm{~m}^{2}$ & $\mathrm{~s}^{-1}$ & - & - \\
\hline \multicolumn{10}{|c|}{ Unidirectional random waves } \\
\hline & 0.0006 & 0.0484 & 0.382 & 3.505 & 0.0023 & 0.0023 & 0.475 & 0.364 & 0.979 \\
\hline \multicolumn{10}{|c|}{ Multi-directional random waves ( 20-degree spreading angle ) } \\
\hline & -0.0055 & 0.0164 & -0.069 & 2.921 & 0.0027 & 0.0029 & 0.535 & 0.539 & 0.989 \\
\hline \multicolumn{10}{|c|}{ Random waves and a current of $0.244 \mathrm{~m} / \mathrm{s}$} \\
\hline Test \# 1 & -0.122 & 0.071 & -0.156 & 2.431 & 0.0199 & 0.0181 & 0.150 & 0.820 & 0.997 \\
\hline Test \# 2 & -0.115 & 0.052 & -0.330 & 2.887 & 0.0159 & 0.0144 & 0.097 & 0.867 & 0.999 \\
\hline Test \# 3 & -0.117 & 0.054 & -0.738 & 3.329 & 0.0165 & 0.0159 & 0.102 & 0.877 & 0.999 \\
\hline Test \# 4 & -0.110 & 0.055 & -0.692 & 3.418 & 0.0152 & 0.0144 & 0.094 & 0.877 & 0.999 \\
\hline \multicolumn{10}{|c|}{ Random waves and a current of $0.366 \mathrm{~m} / \mathrm{s}$} \\
\hline Test \# 1 & -0.273 & 0.0678 & -0.339 & 2.787 & 0.0790 & 0.0708 & 0.091 & 0.822 & 1.000 \\
\hline Test \# 2 & -0.250 & 0.0682 & -0.032 & 1.989 & 0.0671 & 0.0605 & 0.098 & 0.829 & 1.000 \\
\hline Test \# 3 & -0.280 & 0.0512 & 0.195 & 2.584 & 0.0812 & 0.0755 & 0.083 & 0.830 & 1.000 \\
\hline Test \# 4 & -0.276 & 0.0546 & 0.106 & 3.067 & 0.0793 & 0.0729 & 0.083 & 0.836 & 1.000 \\
\hline
\end{tabular}

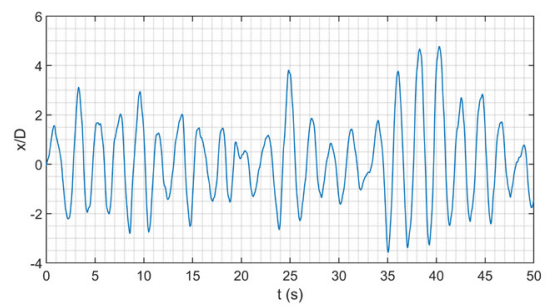

(a)

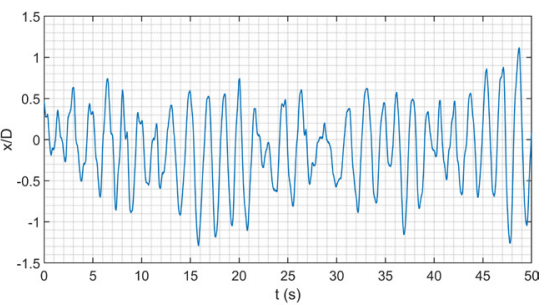

(b)

Figure 10: Zoomed-in plot of $X(t)$ of (a) unidirectional random waves; (b) multi-directional random waves.

this problem, the separate four tests under each flow condition with a current were combined for the analysis.

The particulars of the GEV statistical models selected for each test conditions were summarized in Table 3, and the corresponding quantile plots were presented in Figs 11 and 12. In Table 3, one observes that when the cylinder was only subjected to random waves, the AD test p-values under the first two cases are substantially above 0.25 , which indicates an excellent fit. This excellent agreement between the model and the data can also be found in Fig. 11, where the predicted model quantiles almost coincide with the observed empirical quantiles on the 45-degree reference line and only a few small outliers were observed in the upper tail. When a steady current of $0.244 \mathrm{~m} / \mathrm{s}$ and of $0.366 \mathrm{~m} / \mathrm{s}$ was, respectively, superposed to the 
Table 3: Particulars of GEV statistical models fitted to the cylinder's flow-induced response amplitudes.

\begin{tabular}{lllllllll}
\hline $\begin{array}{l}\text { Flow } \\
\text { conditions }\end{array}$ & $\begin{array}{l}\text { Block } \\
\text { size } n\end{array}$ & $\begin{array}{l}\text { AD test } \\
\text { p-value }\end{array}$ & $\begin{array}{l}\text { Goodness } \\
\text { of fit }\end{array}$ & $\breve{\xi}$ & $\breve{\mu}$ & $\breve{\sigma}$ & \multicolumn{2}{l}{$\begin{array}{l}\text { Threshold-crossing } \\
\text { probabilities }\end{array}$} \\
\hline $\begin{array}{l}\text { Uni-directional } \\
\text { random waves }\end{array}$ & 80 & 0.394 & Excellent & 0.0363 & 1.5510 & 0.6641 & $P(Z>2 D)$ & $P(Z>4 D)$ \\
& & & & & & & 0.401 & $3.091 \times 10^{-2}$ \\
$\begin{array}{l}\text { Multi-direc- } \\
\text { tional random } \\
\text { waves }\end{array}$ & 80 & 0.526 & Excellent & -0.0992 & 0.6434 & 0.2384 & $P(Z>1 D)$ & $P(Z>2 D)$ \\
$\begin{array}{l}\text { Random waves } \\
\text { and } 0.244 \mathrm{~m} / \mathrm{s} \\
\text { current }\end{array}$ & 80 & 0.088 & $\begin{array}{l}\text { Moderate- } \\
\text { ly good }\end{array}$ & -0.1835 & 4.4272 & 1.2333 & $P(Z>8 D)$ & $P(Z>10 D)$ \\
$\begin{array}{l}\text { Random waves } \\
\text { and } 0.366 \mathrm{~m} / \mathrm{s} \\
\text { current }\end{array}$ & 80 & 0.186 & Good & -0.1759 & 8.7552 & 0.9167 & $P(Z>10 D) P(Z>12 D)$ \\
\hline
\end{tabular}

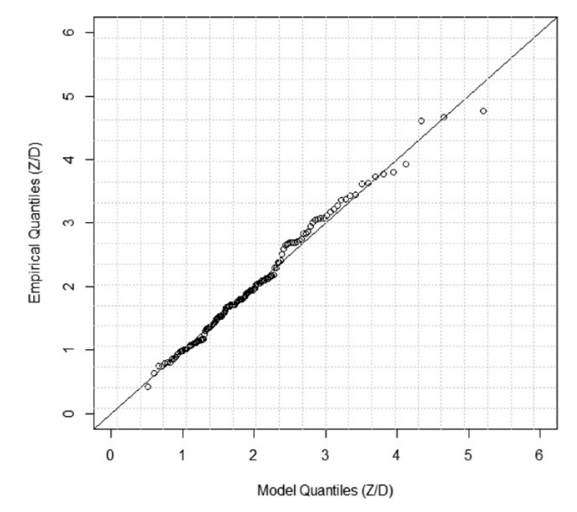

(a)

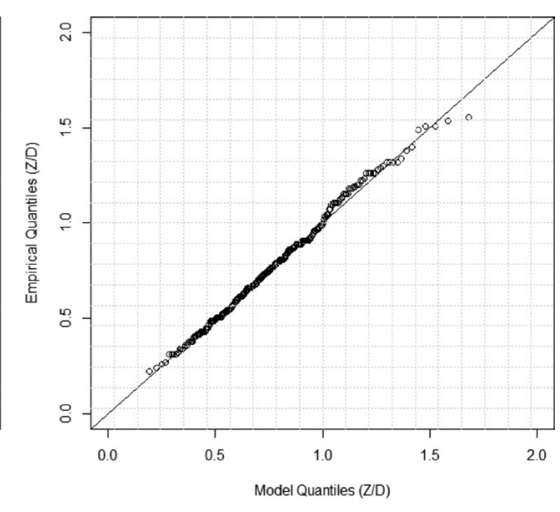

(b)

Figure 11: Quantile plot of the GEV model fitted to the maximum amplitude of the cylinder's displacement when subject to (a) unidirectional random waves and (b) multidirectional random waves.

random waves, the AD test $\mathrm{p}$-values decreased significantly but were still above 0.05 . Further, in Fig. 12, one observes that although there are noticeable discrepancies between the model and the data, the data still follow the trend of the 45-degree reference line and the deviations remain small. Thus, the GEV statistical model could give an at least moderately good fit to this data set. Based on the selected GEV models, the threshold-crossing probabilities of the observed data could be assessed, and two predicted values were given in Table 3 for illustration. The threshold values were determined based on the maximum observed mid-span displacement $X(t)$. For the combined wave and current excitations, significantly larger values were chosen for the thresholds to account for the mean deflection due to the current-induced 


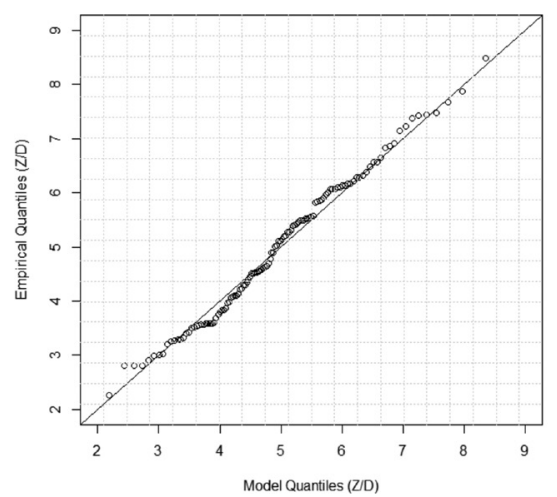

(a)

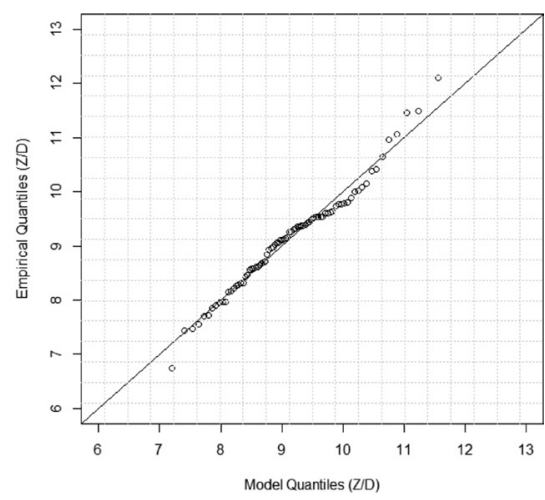

(b)

Figure 12: Quantile plot of the GEV model fitted to the maximum displacement in-line with the current direction for a cylinder subject to random waves and a current of (a) $0.244 \mathrm{~m} / \mathrm{s}$ and (b) $0.366 \mathrm{~m} / \mathrm{s}$.

sag. These findings are noteworthy considering the complexity of the flexible cylinder's flow-induced response behavior when subjected to combined random waves and a strong current. The GEV statistical model is found capable of capturing the statistical characteristics of the extreme values observed in the cylinder's flow-induced response amplitude obtained in this model basin test program [13].

\section{SUMMARY AND CONCLUSIONS}

This article presents a general approach to investigate the statistical characteristics of flexible horizontal cylinder's flow-induced response amplitude observed in a model basin test program [13]. The cylinder was tested under a combination of random waves and current loadings. Spectral analysis was first performed to the measured cylinder's mid-span displacement $X(t)$, of which both the spectral and the statistical moments were calculated to interpret the cylinder's response behavior. In the next phase of the analysis, a statistical approach based on extremal statistics methods was introduced. This approach requires the subdivision of the time series into a sequence of block maxima $\left\{Z_{n, i}\right\}$ to extract the extreme values in the measured data $X(t)$. The generalized extreme value (GEV) distribution was fitted to the sequence $\left\{Z_{n, i}\right\}$, and the model's performance was assessed by both the Anderson-Darling (AD) test criterion and the use of quantile plots.

For the case when the cylinder was only subject to random waves, the mid-span displacement $X(t)$ was found to be weakly non-Gaussian. When a steady current was superposed on the random wave excitation, the third and fourth statistical moments illustrated significant variations among different tests even under the same flow condition, while the expected VIV frequencies were completely absent in the corresponding PSD plots. These observations suggest that the flow-induced response behavior is too complicated to be adequately characterized by a set of deterministic parameters. These observations are consistent with the earlier research finding of Resvanis and Vandiver [12], who concluded that the VIV response of flexible cylinders was stochastic in nature even in a steady flow, and they called for further research investigation into the observed response variability. A noteworthy finding is that the GEV distribution provides an excellent fit when the cylinder was subject to random waves alone, and it could at least provide a moderately good fit even with a strong current 
superposed to the random waves. These research findings based on the OTRC model basin data [13] indicate that the extremal methodology presented could be useful to capture the essential characteristics of the stochastic flow-induced response behavior. This methodology enables engineers to utilize the information contained in the whole data set instead of trying to characterize a complex fluid-structure interaction with just a few deterministic parameters. It provides a means to choose the most appropriate statistical model for a specific design scenario. Having made the selection of the most appropriate extremal statistics model, engineers would be able to make probabilistic predictions of exceedance over different design conditions for the system. The methodology is promising and merits further investigation of other laboratory and field data.

\section{ACKNOWLEDGEMENTS}

The authors gratefully acknowledge the partial financial support of the Cain Senior Chair endowment for Offshore Technology during this research study. The senior author dedicates this article to the memory of Dr. Carlos Brebbia, a friend and scholar whom we lost all too soon.

\section{REFERENCES}

[1] Vandiver, J.K., Dimensionless parameters important to the prediction of vortex-induced vibration of long, flexible cylinders in ocean currents. Journal of Fluids and Structures, 7(5), pp. 423-455, 1993. https://doi.org/10.1006/jfls.1993.1028

[2] Griffin, O.M., Skop, R.A. \& Ramberg, S.E., Resonant, Vortex-Excited Vibrations of Structures and Cable Systems. Offshore Technology Conference, Houston, 1975.

[3] Sarpkaya, T., Vortex induced oscillations. Journal of Applied Mechanics, 46(2), pp. 241-258, 1979. https://doi.org/10.1115/1.3424537

[4] Sarpkaya, T., A critical review of the intrinsic nature of VIV, Journal of Fluids and Structures, 19(4), pp. 389-447, 2004.

[5] Zdravkovich, M.M., On origins of hysteretic responses of a circular cylinder induced by vortex shedding. Zeitschrift fur Flugwissenschaften und Weltraumforschung (Journal of Flight Sciences and Space Research), 14, pp. 47-58. Springer-Verlag, 1990.

[6] Vandiver, J.K., Damping parameters for flow-induced vibration. Journal of Fluids and Structures, 35, pp. 105-119, 2012. https://doi.org/10.1016/j.jfluidstructs.2012.07.002

[7] Klamo, J.T., Leonard, A. \& Roshko, A., On the Maximum Amplitude of a Freely Vibrating Cylinder in Cross-Flow. Journal of Fluids and Structures, 21(4), pp. 429-434, 2005. https://doi.org/10.1016/j.jfluidstructs.2005.07.010

[8] Swithenbank, S.B., Vandiver, J.K., Larsen, C.M. \& Lie, H., Reynolds Number Dependence of Flexible Cylinder VIV Response Data. Proceedings of the International Conference on Offshore Mechanics and Artic Engineering, OMAE2008-57045, 2008.

[9] Resvanis, T.L., Jhingran, V., Vandiver, J.K. \& Liapis, S., Reynolds number effects on the vortex-induced vibration of flexible marine risers. Proceedings of the ASME 2012 31st International Conference on Ocean, Offshore and Arctic Engineering, OMAE 2012-83565, 2012.

[10] Govardhan, R.N. \& Williamson, C.H.K., Defining the Modified Griffin Plot in Vortex-Induced Vibration: Revealing the Effect of Reynolds Number Using Controlled Damping. Journal of Fluid Mechanics, 561, 147-180, 2006. https://doi.org/10.1017/ s0022112006000310

[11] MARINTEK, Shell Riser VIV Tests Main Report, No. 580233.00.0, 2011. 
[12] Resvanis, T.L. \& Vandiver, J.K., Response variability in flexible cylinder VIV model test data. Proceedings of the ASME 2017 36th International Conference on Ocean, Offshore and Arctic Engineering, OMAE 2017-61516, 2017.

[13] Chitwood, J.S., Vortex-induced vibration of a slender horizontal cylinder in currents and waves, OTRC report No. 2/98-A9575, 1998.

[14] Nigam, Introduction to Random Vibrations. The MIT Press, Cambridge, MA, 1983.

[15] Cartwright, D.E. \& Longuet-Higgins M.S., The statistical distribution of the maxima of a random function. Proc Royal Soc Lond A, 237:212-232, 1956. https://doi. org/10.1098/rspa.1956.0173

[16] Coles S., An introduction to statistical modeling of extreme values. Spinger, Verlag London, 2001.

[17] D’Agostino, R.B. \& Stephens, M.A., eds., Goodness-of-fit techniques. Marcel Dekker, New York, 1986.

[18] Cramér H., On the composition of elementary errors. Skand. Aktuarietids, 11, pp. 13-74, 141-180, 1928.

[19] Anderson, T.W. \& Darling, D.A., A test for goodness of fit. Journal of the American Statistical Association, 49(268), pp. 765-769, 1954. https://doi.org/10.1080/0162145 9.1954.10501232

[20] Welch, P.D., The use of Fast Fourier Transform for the estimation of power spectra: A method based on time averaging over short, modified periodograms. IEEE Transactions on Audio and Electroacoustics, AU-15(2), 70-73, 1967. https://doi.org/10.1109/ tau.1967.1161901

[21] R Core Team, R: A language and environment for statistical computing. $R$ Foundation for Statistical Computing, Vienna, Austria, 2019. URL https://www.R-project.org/ 